\title{
Krank durchs Krankenhaus?
}

\author{
Hygienemängel und Behandlungsfehler - Krankenhäuser sind eigentlich Orte der \\ Heilung. Und doch ist manchmal genau das Gegenteil der Fall: Man kommt kränker heraus, \\ als man hinein gegangen ist. Das Landgericht München I hatte sich mit zwei derartigen \\ Streitfällen zu befassen und verkündete am 27.8.2008 zwei ganz unterschiedliche Urteile.
}

$>$

Die Klägerin im ersten Rechtsstreit (Az: 9 O 13805/05) hatte sich 2004 in einer Münchner Klinik einer Darmoperation unterzogen. Kaum hatte sie das Krankenhaus verlassen, wurde bei ihr eine akute Hepatitis-C-Infektion festgestellt. Sie verklagte darauf die Klinik auf Schadensersatz mit der Begründung, dass die Infektion durch mangelnde Hygiene in der Klinik verursacht worden sei. Nach dem Gutachten des gerichtlich bestellten Sachverständigen hatte sich die Klägerin tatsächlich dort infiziert. Dennoch sah das Landgericht kein schuldhaftes Fehlverhalten der Klinik: Laut Gutachter lasse das Auftreten einer Infektion noch keinen Schluss auf die Verletzung hygie-

\section{(2) \\ - HABEN SIE NOCH FRAGEN?}

Rolf Höfert, Geschäftsführer des Deutschen Pflegeverbandes (DPV), Autor des Buches „Von Fall zu Fall", Experte für Pflegerecht und Mitbegründer des jährlichen Heilberufe-Pflege-RechtTages, ist im Beirat der Redaktion für die Rubrik „Pflegerecht" verantwortlich. Gern greift er Ihre Fragen für diese Seiten auf.

Senden Sie diese am besten per Email an: heilberufe@urban-vogel.de nischer Standards zu. In der Medizin sei es nach wie vor rätselhaft, auf welchem Wege, außer etwa durch Blutkontakt, Hepatitis-C übertragen werde. Auch in diesem Fall konnte der Übertragungsweg nicht geklärt werden. Das Landgericht konnte daher auch nicht feststellen, dass das Infektionsrisiko für die Klinik voll beherrschbar war. Auch eine bei den Gesundheitsbehörden eingeholte Auskunft ergab keine Anhaltspunkte für eine Infektion beim Krankenhauspersonal. Die Klage wurde daher abgewiesen.

\section{Fataler Behandlungsfehler}

Im anderen Rechtsstreit (Az: 9 O 11016/ $05)$ hatte der mittlerweile verstorbene

Sohn des Klägers eine Münchner Klinik wegen einer HNO-Operation aufgesucht. Die Operation war fast beendet, als der Patient zur postoperativen Schmerzausschaltung ein Medikament gespritzt bekam - was zu einer Tragödie führte.

Der bekanntermaßen an Asthma leidende Patient reagierte auf das Medikament mit einem Bronchospasmus. Das Gehirn des Patienten wurde für einige Minuten nur unzureichend mit Sauerstoff versorgt. Zwar besserte sich der Zustand des Patienten zunächst. Er blieb aber auch im Aufwachraum bewusstlos und erlitt letztlich eine Hirnschädigung. Unklar ist, was im Verlauf einer ganzen Stunde im Aufwachraum passierte: Es fehlt jede Aufzeichnung für diesen Zeitraum. Das Gericht stellte nach Anhörung eines Sachverständigen einen groben Behandlungsfehler fest: Das Medikament sei nicht nur zu hoch dosiert gewesen, es hätte angesichts der Asthmaerkrankung gar nicht verabreicht werden dürfen. Im Aufwachraum befand sich der Patient in einem massiven Schockzustand, ohne dass eine adäquate Reaktion dokumentiert wurde. Die Klinik wurde daher zur Zahlung von Schmerzensgeld verurteilt, über dessen Höhe noch zu entscheiden ist.

- Werner Schell

320 S., 25 Abb., brosch. 19,95€ ISBN 978-3-54079328-1

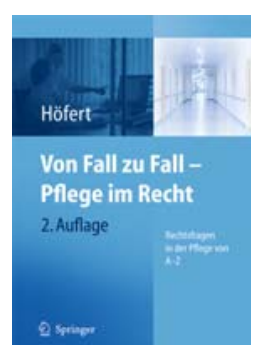

Bitte beachten Sie! Heilberufe übernimmt keinerlei Gewähr für die Aktualität, Korrektheit, Vollständigkeit oder Qualität der bereitgestellten Informationen. Haftungsansprüche gegen Heilberufe, die sich auf Schäden materieller oder ideeller Art beziehen, die durch die Nutzung oder Nichtnutzung der dargebotenen Informationen beziehungsweise durch die Nutzung fehlerhafter und unvollständiger Informationen verursacht wurden, sind grundsätzlich ausgeschlossen. 